\title{
Expression of ribonucleoside reductase subunit M1, but not excision repair cross-complementation group 1, is predictive in muscle-invasive bladder cancer treated with chemotherapy and radiation
}

\author{
MARK SHILKRUT $^{1 *}$, ANGELA WU $^{2 *}$, DAFYDD G. THOMAS $^{2}$ and DANIEL A. HAMSTRA ${ }^{3}$ \\ ${ }^{1}$ Department of Oncology, Rambam Health Care Campus, Haifa 31096, Israel; \\ Departments of ${ }^{2}$ Pathology and ${ }^{3}$ Radiation Oncology, University of Michigan, Ann Arbor, MI 48109, USA
}

Received December 26, 2013; Accepted February 13, 2014

DOI: $10.3892 / \mathrm{mco} .2014 .264$

\begin{abstract}
The aim of this study was to determine the prognostic and predictive values of ribonucleoside reductase subunit M1 (RRM1) and excision repair cross-complementation group 1 (ERCC1) expression in patients with muscle-invasive bladder cancer treated with chemoradiotherapy. The expression of RRM1 and ERCC1 in pretreatment tumor samples of retrospectively identified patients was determined by immunohistochemical analysis. A total of 39 patients were included in this study; $49 \%$ were treated with neoadjuvant chemotherapy and $67 \%$ with concomitant chemoradiotherapy; $56 \%$ were treated with gemcitabine-based and $51 \%$ with platinum-based chemoradiotherapy. The median follow-up was 19 months (interquartile range, 11-50 months). Based on the immunohistochemical analysis, 44 and $32 \%$ of the tumors exhibited increased expression of RRM1 and ERCC1, respectively. The complete response (CR) and local recurrence rates following chemoradiotherapy were 79 and $21 \%$, respectively. A low expression of RRM1 was associated with a higher rate of $\mathrm{CR}$ to chemoradiotherapy (95 vs. $57 \%, \mathrm{P}=0.012$ ); however, there was no such association with low ERCC1 expression (67 vs. $84 \%, \mathrm{P}=0.39$ ). RRM1 expression predicted an improved $\mathrm{CR}$ in patients treated with gemcitabine-based chemoradiotherapy (57 vs. $100 \%, \mathrm{P}=0.036$ ), but not in those treated with other agents (56 vs. $88 \%, \mathrm{P}=0.29)$. ERCC1 expression was not found to be correlated with CR (67 vs. $84 \%, \mathrm{P}=0.39$ ), even when restricted to patients treated with platinum
\end{abstract}

Correspondence to: Dr Mark Shilkrut, Department of Oncology, Rambam Health Care Campus, PO Box 9602, Haifa 31096, Israel

E-mail: mark.shilkrut@gmail.com

*Contributed equally

Key words: bladder cancer, ribonucleoside reductase subunit M1, excision repair cross-complementation group 1, radiotherapy, chemotherapy, prognostic marker agents ( 71 vs. $75 \%, P=1.0$ ). In the univariate analysis, RRM1 expression, but not ERCC1 expression, was identified as a prognostic marker for worse cancer-specific survival in all the patients and in those treated with gemcitabine-based regimens. No independent prognostic factor was identified in the multivariate model, which included tumor stage, vascular invasion, hydronephrosis and RRM1 status. Although these findings require further validation, they suggest that RRM1 may be a beneficial stratification variable for the selection of chemotherapy regimens for chemoradiotherapy, with patients with low RRM1 expression being considered suitable for gemcitabine treatment.

\section{Introduction}

Muscle-invasive bladder cancer (MIBC), which constitutes $30 \%$ of all bladder cancers (BCs), is responsible for an annual death toll of $\sim 15,000$ individuals in the United States (1). A viable treatment option for MIBC includes trimodality therapy with a combination of transurethral bladder resection (TURBT), chemotherapy and radiation (with cystectomy for failures), although this method has never been compared to radical cystectomy in randomized clinical trials. This type of treatment has achieved cure rates comparable to extirpative surgery, while preserving a functional bladder (2-4). This combined-modality approach is also a treatment of choice for several patients who may be unsuitable for surgery.

Chemotherapy administered either sequentially or concomitantly with radiation is crucial for organ preservation in $\mathrm{BC}$ by improving local effectiveness (5-7) and increasing survival (7). When administered as systemic agents for BC, platinum- and/or gemcitabine-based regimens are effective in metastatic disease and are also increasingly utilized in the neoadjuvant and adjuvant settings (8). However, the selection of the most appropriate chemotherapy regimen varies, with certain physicians favoring cisplatin-based regimens, such as methotrexate, vinblastine, doxorubicin and cisplatin, or cisplatin, methotrexate and vinblastine, due to their long history of use, whereas others favor gemcitabine-based regimens, which are more tolerable (8). Molecular markers that may aid the selection of appropriate chemotherapy regimens are currently 
under investigation; however, the number of available studies investigating the molecular prognostic markers in trimodality therapy is currently limited.

Ribonucleoside reductase subunit M1 (RRM1), a substrate-binding and regulatory subunit of ribonucleotide reductase, is the primary cellular target of gemcitabine (9). The overexpression of RRM1 was implicated in resistance to gemcitabine in various tumor cell lines and models (9-11). Excision repair cross-complementation group 1 (ERCC1) is a key part of the nucleotide-excision repair pathway that removes platinum-DNA adducts and counteracts the cytotoxic effects of platinum agents (12). ERCC1 expression was reported to confer resistance to platinum derivatives and has been evaluated as a predictive and prognostic marker in various tumors, where platinum is the cornerstone of chemotherapy (12-17). ERCC1 was also implicated in repairing DNA double-strand breaks and conferring resistance to ionizing radiation, which may help identify the patients that are most suitable to undergo radical cystectomy compared to trimodality therapy $(15,18)$.

The aim of this study was to test the hypothesis that RRM1 and ERCC1 expression predict tumor response and clinical outcome in patients with MIBC treated with combined platinum- or gemcitabine-based chemotherapy and radiation.

\section{Materials and methods}

Patients and treatment. An institutional review board-approved study was undertaken on patients treated with chemoradiotherapy for MIBC in the University of Michigan (Ann Arbor, MI, USA) between 1999 and 2012. A total of 83 patients with MIBC were identified, among whom 39 had pretreatment tumor samples suitable for the construction of tissue microarrays (TMAs). There was no uniform treatment protocol for MIBC; thus, the patients were treated with either neoadjuvant polychemotherapy followed by radiation or with concomitant chemoradiotherapy from the beginning. Prior to treatment, all the patients underwent cystoscopy, TURBT and abdominopelvic and chest imaging by computed tomography (CT). Following treatment and during follow-up, the patients underwent periodic cystoscopy and CT every 3-4 months for the first 2 years and less frequently thereafter.

For radiotherapy planning, all the patients underwent CT-based simulation. The target volume included either the bladder alone with a margin of $\geq 1.5-\mathrm{cm}$, or the bladder with margins and the pelvic lymph nodes. The prescribed dose to the bladder was 59.4-64.8 Gy and to the pelvis 39.6-50 Gy. Radiation was delivered in 1.8- to 2-Gy daily fractions using $\geq 6 \mathrm{MV}$ photons. For the statistical analysis, all the fractions were converted into $2-G y$ equivalent dose using an $\alpha / \beta$ ratio of 10 .

Immunohistochemical analysis of ERCCI and RRM1 expression. There is currently no standard method for the evaluation of ERCC1 and RRM1 expression; thus, immunohistochemical analysis and quantitative reverse transcription-polymerase chain reaction (qRT-PCR) were previously used to determine ERCC1 and RRM1 expression. The decision to use immunostaining in our study was based on a previous study reporting that ERCC1 and RRM1 mRNA measurements by qRT-PCR were not correlated with immunohistochemical findings and that immunostaining was better correlated with survival parameters compared to mRNA measurements in non-small-cell lung cancer (NSCLC) patients (16). TMAs were constructed from the most representative non-necrotic areas of the formalin-fixed, paraffin-embedded tissue blocks (19). Immunohistochemical analysis was performed on the Dako Autostainer (Dako, Carpinteria, CA, USA) using a Dako LSAB+ kit and diaminobenzidine as the chromogen. Serial sections of deparaffinized TMA were labeled for RRM1 (goat polyclonal antibody, 1:100, SC-11731; Santa Cruz Biotechnology, Inc., Santa Cruz, CA, USA) and ERCC1 (mouse monoclonal antibody, 8F1, 1:200, MS-671; Neomarkers, Inc., Fremont, CA, USA). Microwave citric acid epitope retrieval was used for the two antibodies. Appropriate negative (no primary antibody) and positive control cores (breast cancer tissue for RRM1 and tonsillar tissue for ERCC1) were included in the construction of the TMA. Each core on the TMA was assessed for RRM1 and ERCC1 expression by a genitourinary pathologist who was blinded to the outcomes. An H-score of 0-300 was calculated for both markers by multiplying the staining intensity of the tumor cells ( 0 , no staining; 1 , weak; 2 , moderate; and 3 , strong staining) with the percentage of stained tumor cells in the TMA core (0-100\%). For RRM1, nuclear and/or cytoplasmic staining was considered, while for ERCC1 only nuclear staining was considered. The final $\mathrm{H}$-score was the average score obtained from two 1-mm diameter cores that represented each tumor sample. For ERCC1, tumors with an $\mathrm{H}$-score higher than the median were considered to be expressing the marker $(13,14)$. The receiver operating characteristic (ROC) curve analysis was then used to determine the cutoff point between tumors with high or low expression of RRM1, as the former was better correlated with distant metastasis (DM) and cancer-specific survival (CSS) compared to the median value or the semi-quantitative $\mathrm{H}$-score.

Treatment outcomes. Complete response (CR) was defined as no evidence of BC, including carcinoma in situ (CIS) and papillary $\mathrm{BC}(\mathrm{Ta})$, documented by cystoscopy and body imaging following completion of all therapies. Local recurrence (LR) was defined as histologically documented reappearance of BC, including CIS and Ta, in the bladder and/or irradiated volume following documented CR to chemoradiotherapy. DM was defined as any clinical, radiographic or histological evidence of metastasis and BC-specific death (BCSD) was defined as death from $\mathrm{BC}$ or from any cause following the development of DM. Freedom from LR (FFLR), freedom from DM (FFDM) and CSS were calculated from the initiation of the treatment to the date of LR, DM or BCSD, respectively.

Statistical analysis. The clinical and treatment characteristics of the patients were compared by analysis of variance for continuous variables and the $\chi^{2}$ test for categorical variables. The Kaplan-Meier method and log-rank test were used for the univariate analysis. A multivariate analysis (MVA) was performed using the Cox proportional hazards regression model. All the statistical analyses, including ROC curve analysis, were performed with MedCalc v.12 software (MedCalc, Ostend, Belgium). $\mathrm{P}<0.05$ was considered to indicate a statistically significant difference. 


\section{Results}

Patient characteristics and clinical outcome. The clinical characteristics and treatment details of 39 patients with MIBC treated with chemoradiotherapy are presented in Table I. All the tumors were high-grade transitional cell urothelial carcinomas. A total of 4 patients had T4 tumors and 3 had positive pelvic lymph nodes (stage IV). The median follow-up was 19 months [interquartile range (IQR), 11-50 months].

The most common neoadjuvant agent was platinum (cisplatin or carboplatin; $n=18)$ followed by gemcitabine $(n=14)$ and paclitaxel $(\mathrm{n}=12)$, with some patients receiving combination regimens with $2(n=6)$ or $3(n=10)$ agents. The most common neoadjuvant regimen was carboplatin-gemcitabine-paclitaxel $(n=8)(20)$, followed by cisplatin-gemcitabine $(n=5)$, carboplatin-paclitaxel $(n=3)$, cisplatin-etoposide $(n=2)$ and gemcitabine-paclitaxel $(n=1)$. The most common concomitant agents administered during radiotherapy were fluoropyrimidines (5-fluorouracil or capecitabine) $(n=13)(21)$ followed by biweekly gemcitabine $(n=18)(22)$ and platinum $(n=7)$ (data not shown).

The rate of $\mathrm{CR}$ at the end of the treatment was $79 \%$, although no patients underwent immediate cystectomy, even with incomplete response. LR occurred in 8 patients (21\%) following CR at the median time of 9 months (IQR, 7-12 months) after the completion of radiotherapy. Salvage cystectomy was performed in 2 patients at 11 and 64 months after the completion of chemoradiotherapy. DM was observed in $28 \%$ of patients; among those with LR, 2 patients with invasive LR (25\%) developed DM thereafter. The crude rate of BCSDs ( $=12 ; 31 \%)$ marginally outnumbered DM $(n=11 ; 28 \%)$, since 1 patient succumbed to the complications of a locally advanced tumor.

FFLR, FFDM and CSS in all the patients and in those grouped by tumor stage are illustrated in Fig. 1 . There was a significant difference in FFDM $(\mathrm{P}=0.015)$ and CSS $(\mathrm{P}=0.017)$ between the various tumor stages, but not in FFLR $(\mathrm{P}=0.71)$. Similarly, there were differences in FFDM and CSS, but not in FFLR, when the patients were grouped by the presence or absence of pretreatment hydronephrosis [hazard ratio $(\mathrm{HR})=3.5,95 \%$ confidence interval $(\mathrm{CI}): 0.52-23.54$; $\mathrm{P}=0.20$ for FFLR; HR=5.4, 95\% CI: $1.17-25.20 ; \mathrm{P}=0.001$ for FFDM; and $\mathrm{HR}=5.29,95 \% \mathrm{CI}$ : $1.18-23.73 ; \mathrm{P}=0.030$ for $\mathrm{CSS}]$ or vascular invasion $(\mathrm{VI})(\mathrm{HR}=1.17,95 \% \mathrm{CI}$ : $0.13-10.79 ; \mathrm{P}=0.88$ for FFLR; HR=10.21, 95\% CI: 1.96-53.15; $\mathrm{P}=0.006$ for FFDM; and $\mathrm{HR}=2.80,95 \% \mathrm{CI}: 0.58-13.58 ; \mathrm{P}=0.076$ for $\mathrm{CSS}$ ). There were no such differences when the patients were grouped by the presence or absence of CIS (HR=0.95, 95\% CI: 0.23-3.95; $\mathrm{P}=0.95$ for FFLR; $\mathrm{HR}=0.30,95 \% \mathrm{CI}: 0.28-3.23 ; \mathrm{P}=0.94$ for FFDM; and $\mathrm{HR}=0.86$, 95\% CI: $0.27-2.78 ; \mathrm{P}=0.80$ for $\mathrm{CSS}$ ) (data not shown).

Association between RRMI and ERCC1 status and clinical outcome. Among the 39 tumor samples available, 36 were successfully processed for RRM1 and 37 for ERCC1 analysis (Fig. 2). The median H-score was 50 (IQR, 0-100) for the RRM1 and 10 (IQR, 0-60) for the ERCC1 staining. The cutoff point for high expression of RRM1 at an H-score of $>60$ was determined by a non-biased ROC curve, with a specificity and sensitivity of 72.7 and $64.0 \%$ for the BCSD, respectively
Table I. Clinical characteristics and treatment outcomes of 39 patients with transitional cell carcinoma of the bladder.

\begin{tabular}{|c|c|}
\hline Characteristics & No. $(\%)$ \\
\hline Median age, years (IQR) & $72(67-80)$ \\
\hline Median follow-up, months (IQR) & $19(11-50)$ \\
\hline \multicolumn{2}{|l|}{ Histology } \\
\hline Aberrant differentiation & $11(28)$ \\
\hline Adjacent carcinoma in situ & $16(41)$ \\
\hline Vascular invasion & $7(18)$ \\
\hline Hydronephrosis & $10(26)$ \\
\hline \multicolumn{2}{|l|}{ Clinical stage $^{a}$} \\
\hline II & $21(54)$ \\
\hline III & $15(38)$ \\
\hline IV $(\mathrm{N}+)$ & $3(8)$ \\
\hline $\begin{array}{l}\text { Median prescribed dose of } \\
\text { radiation, 2-Gy equivalent (IQR) }\end{array}$ & $\begin{array}{c}60.0 \\
(60.0-64.0)\end{array}$ \\
\hline \multicolumn{2}{|l|}{ Chemotherapy } \\
\hline Neoadjuvant & $19(49)$ \\
\hline Concomitant & $26(67)$ \\
\hline \multicolumn{2}{|l|}{ Chemotherapy regimens } \\
\hline Gemcitabine & $22(56)^{b}$ \\
\hline Platinum agents & $20(51)^{\mathrm{b}}$ \\
\hline Fluoropyrimidines & $13(33)$ \\
\hline Paclitaxel & $12(31)$ \\
\hline \multicolumn{2}{|l|}{ Response to therapy } \\
\hline Complete response & $31(79)$ \\
\hline Treated with gemcitabine $(n=22)$ & $19(86)$ \\
\hline Treated with platinum agent $(n=20)$ & $15(75)$ \\
\hline Local recurrence & $8(21)$ \\
\hline Non MP-invasive carcinoma (Ta, T1) & 3 \\
\hline Carcinoma in situ (Tis) & 1 \\
\hline MP-invasive carcinoma (T2-4) & 4 \\
\hline Distant metastasis & $11(28)$ \\
\hline Total deaths & $22(61)$ \\
\hline Bladder cancer deaths & $12(31)$ \\
\hline
\end{tabular}

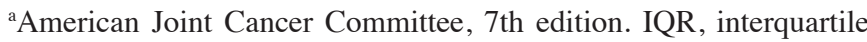

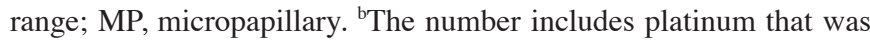
administered concomitantly with radiation and not only neoadjuvant.

$[\mathrm{P}=0.038$, area under the curve $(\mathrm{AUC})=0.71]$, and a specificity and sensitivity of 70.0 and $61.5 \%$ for $\mathrm{DM}$, respectively $(\mathrm{P}=0.12$, AUC $=0.67)$. While the cutoff RRM1 H-score for CR obtained from the ROC curve analysis was $>0(\mathrm{P}=0.0003$, $\mathrm{AUC}=0.78)$, we selected $>60$, as it was associated with clinically meaningful CSS and DM (data not shown) and it was close to the median value, which was utilized as the cutoff score in previous studies $(10,16)$. The ROC curve did not reveal an association between ERCC1 expression and the clinical outcome $(\mathrm{P}=0.39$, $\mathrm{AUC}=0.60$ for $\mathrm{CR} ; \mathrm{P}=0.49, \mathrm{AUC}=0.57$ for $\mathrm{DM}$; and $\mathrm{P}=0.84$, $\mathrm{AUC}=0.52$ for $\mathrm{BC}$ deaths). Therefore, the median $\mathrm{H}$-score 
A
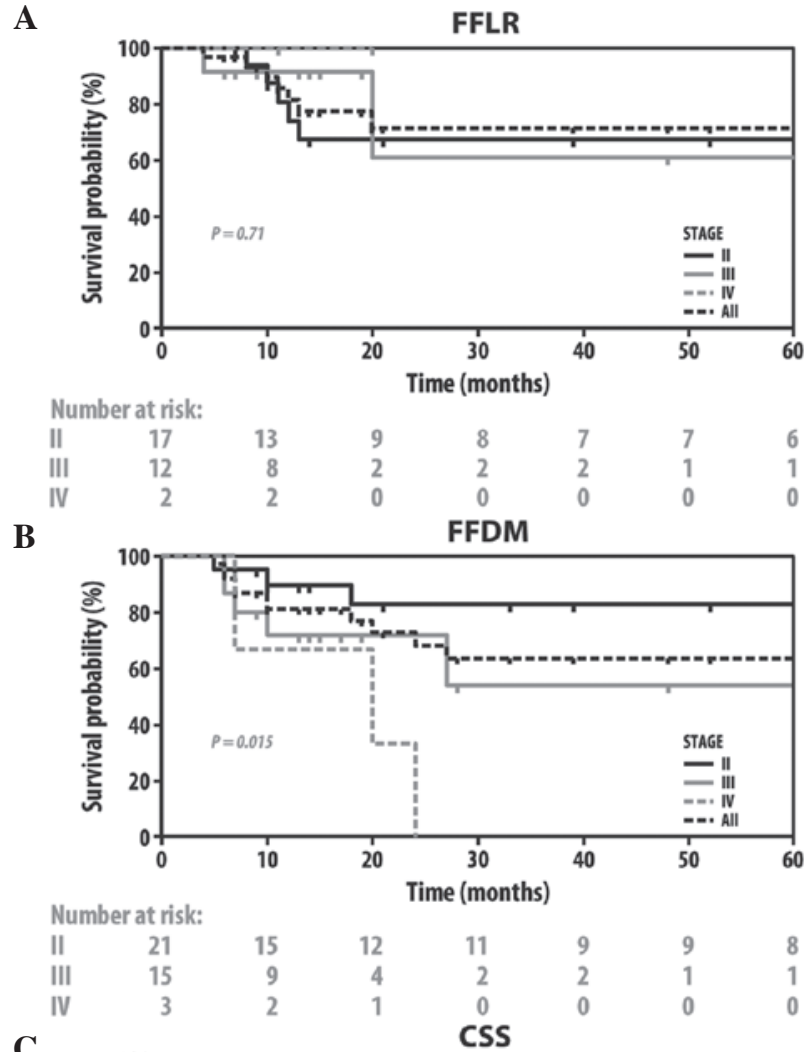

C

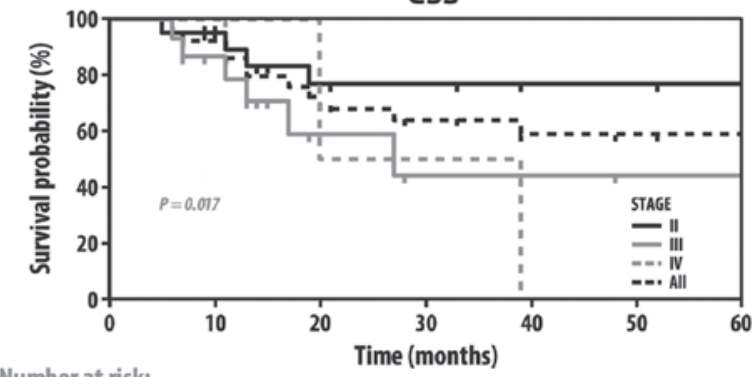

$\begin{array}{lrrrrrrr}\text { II } & 21 & 16 & 12 & 11 & 9 & 9 & 8 \\ \text { III } & 15 & 11 & 4 & 2 & 2 & 1 & 1 \\ \text { IV } & 3 & 3 & 1 & 1 & 0 & 0 & 0\end{array}$

Figure 1. Kaplan-Meier plots of FFLR, FFDM and CSS in all the patients grouped by tumor stage. FFLR, freedom from local recurrence; FFDM, freedom from distant metastasis; CSS, cancer-specific survival.

of $>10$, as previously reported for BC $(13,14)$, was utilized as a cutoff point. Based on these criteria of positivity, 16 patients (44\%) had tumors that highly expressed RRM1, 12 (32\%) had tumors that highly expressed ERCC1, 7 (19\%) had tumors that highly expressed both markers and 15 patients (42\%) had tumors exhibiting low expression of RRM1 and ERCC1. There was no correlation between RRM1 and ERCC1 expression (Spearman's $r_{s}=0.198, P=0.25$ ).

When grouped by RRM1 or ERCC1 status, there were no statistically significant differences between the groups for clinical and treatment characteristics (Table II), except for a trend to a higher rate of hydronephrosis $(\mathrm{P}=0.073)$ in the group highly expressing RRM1. However, following treatment, the tumors exhibiting high expression of RRM1 had a lower rate of CR (56 vs. 95\%, $\mathrm{P}=0.012$ ). When the patients were grouped by whether they received gemcitabine-based chemoradiotherapy or not, there was a statistically significant difference between $\mathrm{CR}$ rates only in the group of patients that received
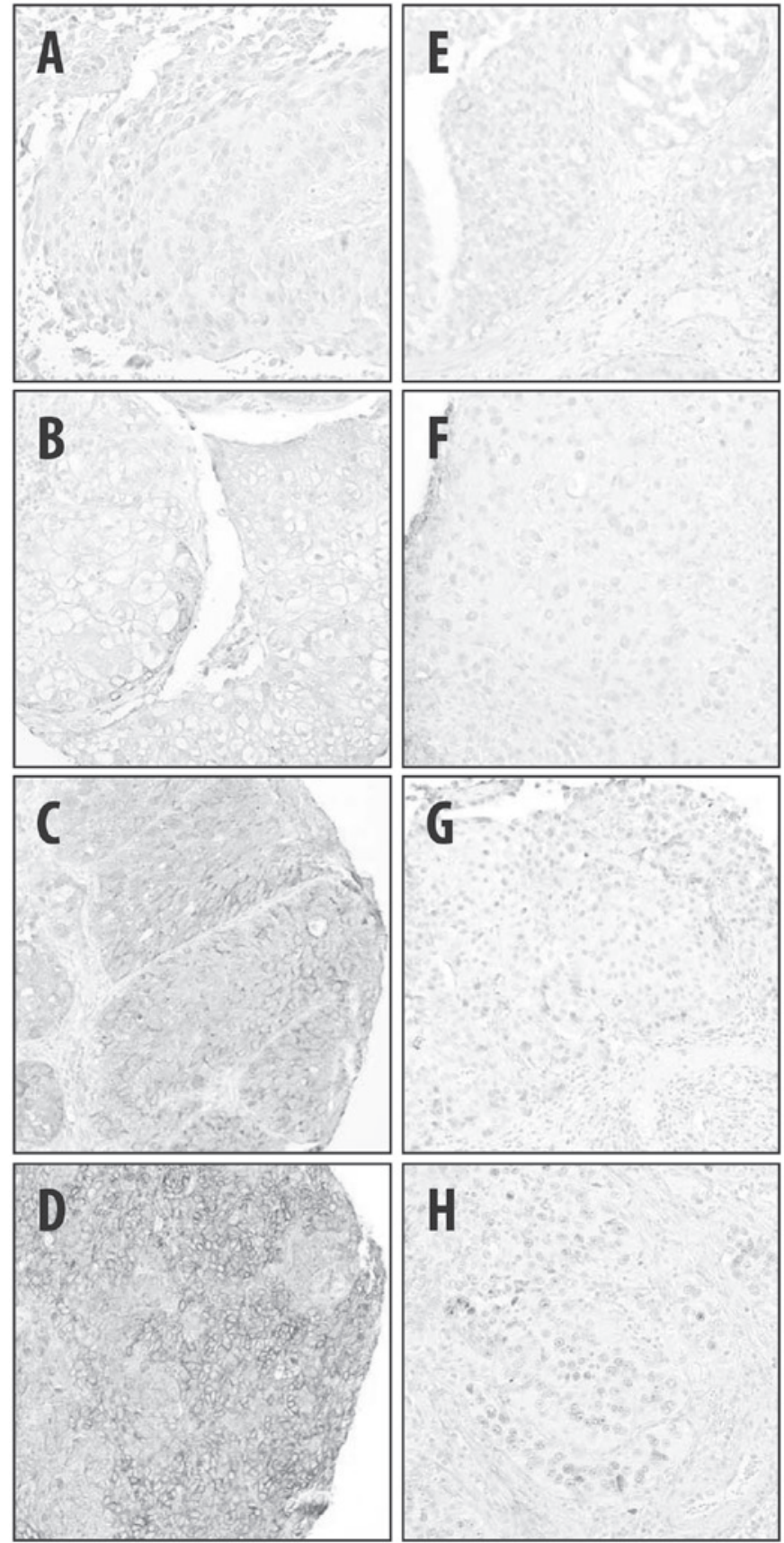

Figure 2. Representative immunohistochemical staining of tumor samples for (A-D) ribonucleoside reductase subunit M1 and (E-H) excision repair cross-complementation group 1 . The staining intensity of the tumor cells was assessed as (A and E) 0, no staining; (B and F) 1, weak; (C and G) 2 , moderate; and $(\mathrm{D}$ and $\mathrm{H}) 3$, strong staining.

gemcitabine ( $\mathrm{P}=0.036$ vs. $\mathrm{P}=0.29$ ) (Table II). The $\mathrm{CR}$ rate was lower in patients with tumors that expressed ERCC1; however, the difference was not statistically significant (67 vs. $84 \%$, $\mathrm{P}=0.39$ ) and there was no LR after attaining $\mathrm{CR}$ in this group of patients. When only platinum-treated patients were considered, the rates of $\mathrm{CR}$ following chemoradiotherapy were indistinguishable between groups based on the ERCC1 status (71 vs. $75 \%, \mathrm{P}=1.0$ ).

Since CR to chemoradiation was shown to be prognostic for later clinical endpoints (23), the effects of RRM1 and ERCC1 expression on other clinically significant endpoints were investigated. The patients with tumors with 
Table II. Clinical characteristics and treatment outcomes of patients grouped by RRM1 and ERCC1 status.

\begin{tabular}{|c|c|c|c|c|c|c|}
\hline \multirow[b]{2}{*}{ Characteristics } & \multicolumn{2}{|c|}{ RRM1 IHC $(n=36)$} & \multirow[b]{2}{*}{$\mathrm{P}$-value } & \multicolumn{2}{|c|}{ ERCC1 IHC $(n=37)$} & \multirow[b]{2}{*}{ P-value } \\
\hline & $\begin{array}{c}\text { High } \\
\mathrm{n}=16(44 \%)\end{array}$ & $\begin{array}{c}\text { Low } \\
n=20(56 \%)\end{array}$ & & $\begin{array}{c}\text { High } \\
\mathrm{n}=12(32 \%)\end{array}$ & $\begin{array}{c}\text { Low } \\
\mathrm{n}=25(68 \%)\end{array}$ & \\
\hline Median age, years (IQR) & $72(69-79)$ & $73(66-81)$ & $0.41^{\mathrm{a}}$ & $75(66-79)$ & $71(67-81)$ & $0.62^{\mathrm{a}}$ \\
\hline Median follow-up, months (IQR) & $14(10-35)$ & $19(11-52)$ & $0.26^{\mathrm{a}}$ & $14(9-24)$ & $20(11-52)$ & $0.22^{\mathrm{a}}$ \\
\hline \multicolumn{7}{|l|}{ Histology } \\
\hline Aberrant differentiation & $6(38)$ & $5(25)$ & $0.48^{\mathrm{b}}$ & $3(25)$ & $8(32)$ & $1.0^{\mathrm{b}}$ \\
\hline Adjacent carcinoma in situ & $8(50)$ & $8(40)$ & $0.74^{\mathrm{b}}$ & $4(16)$ & $10(40)$ & $1.0^{\mathrm{b}}$ \\
\hline Vascular invasion & $5(31)$ & $2(10)$ & $0.20^{\mathrm{b}}$ & $3(25)$ & $4(16)$ & $0.66^{\mathrm{b}}$ \\
\hline Hydronephrosis & $7(44)$ & $3(15)$ & $0.073^{\mathrm{b}}$ & $5(42)$ & $5(20)$ & $0.24^{\mathrm{b}}$ \\
\hline \multicolumn{7}{|l|}{ Clinical stage } \\
\hline II & $7(44)$ & $12(60)$ & $0.50^{\mathrm{b}}$ & $4(33)$ & $16(64)$ & $0.16^{\mathrm{b}}$ \\
\hline III & $7(44)$ & $7(35)$ & $0.72^{\mathrm{b}}$ & $7(58)$ & $7(28)$ & $0.44^{\mathrm{b}}$ \\
\hline IV & $2(13)$ & $1(5)$ & $0.069^{\mathrm{b}}$ & $1(8)$ & $2(7)$ & $0.37^{\mathrm{b}}$ \\
\hline \multicolumn{7}{|l|}{ Chemotherapy } \\
\hline Neoadjuvant & $10(63)$ & $8(40)$ & $0.32^{\mathrm{b}}$ & $7(58)$ & $11(44)$ & $0.50^{\mathrm{b}}$ \\
\hline Concomitant & $10(63)$ & $14(70)$ & $0.73^{\mathrm{b}}$ & $6(50)$ & $19(76)$ & $0.15^{\mathrm{b}}$ \\
\hline \multicolumn{7}{|l|}{ Chemotherapy regimens } \\
\hline Gemcitabine & $7(44)$ & $12(60)$ & $0.50^{\mathrm{b}}$ & $7(58)$ & $13(52)$ & $1.0^{\mathrm{b}}$ \\
\hline Platinum agents & $10(63)$ & $9(45)$ & $0.34^{\mathrm{b}}$ & $7(58)$ & $12(48)$ & $0.73^{\mathrm{b}}$ \\
\hline Fluoropyrimidines & $7(44)$ & $6(30)$ & $0.49^{b}$ & $5(42)$ & $8(32)$ & $0.72^{\mathrm{b}}$ \\
\hline Paclitaxel & $6(38)$ & $5(25)$ & $0.48^{\mathrm{b}}$ & $6(50)$ & $5(20)$ & $0.12^{\mathrm{b}}$ \\
\hline Complete response & $9(56)$ & $19(95)$ & $0.012^{\mathrm{b}}$ & $8(67)$ & $21(84)$ & $0.39^{\mathrm{b}}$ \\
\hline Local recurrence & $2(13)$ & $5(25)$ & $1.0^{\mathrm{b}}$ & 0 & $7(28)$ & $0.142^{\mathrm{b}}$ \\
\hline Distant metastasis & $8(50)$ & $3(15)$ & $0.12^{\mathrm{b}}$ & $2(17)$ & $8(32)$ & $0.56^{\mathrm{b}}$ \\
\hline Total deaths & $14(88)$ & $8(40)$ & $0.078^{\mathrm{b}}$ & $6(50)$ & $14(56)$ & $0.99^{\mathrm{b}}$ \\
\hline Bladder cancer deaths & $9(56)$ & $3(15)$ & $0.057^{\mathrm{b}}$ & $3(25)$ & $8(32)$ & $0.96^{\mathrm{b}}$ \\
\hline
\end{tabular}

${ }^{a}$ Analysis of variance; ${ }^{b} \chi^{2}$ test. RRM1, ribonucleoside reductase subunit M1; ERCC1, excision repair cross-complementation group 1; IHC, immunohistochemistry; IQR, interquartile range.

a low expression of RRM1 exhibited better CSS (HR=3.79, 95\% CI: $1.14-12.59 ; \mathrm{P}=0.033)$ and a trend to FFDM $(\mathrm{HR}=3.4$, 95\% CI: 0.96-11.99; $\mathrm{P}=0.057)$, but no difference in FFLR $(\mathrm{HR}=0.79,95 \% \mathrm{CI}: 0.16-3.77 ; \mathrm{P}=0.77)$ (Fig. 3A-C). There were no such differences when the patients were grouped by ERCC1 status. (Fig. 4A-C). To further investigate the prognostic significance of RRM1 and ERCC1, we evaluated FFDM and CSS when only patients treated with gemcitabine-based chemotherapy were grouped by RRM1 status (Fig. 3D-F) and patients treated with platinum-based chemotherapy were grouped by ERCC1 status (Fig. 4D-F). Consistent with the previous findings of the present study, patients with tumors with a low expression of RRM1 exhibited a better CSS (HR=4.85, 95\%CI: $1.02-23.18 ; \mathrm{P}=0.036)$ and a trend to FFDM (HR=4.21, 95\% CI: 0.76-23.32; $\mathrm{P}=0.070)$ on receiving gemcitabine-based therapy. No such difference was observed in patients with tumors with a high expression of RRM1 who did not receive gemcitabine $(\mathrm{HR}=3.01,95 \% \mathrm{CI}$ : $0.42-21.4$; $\mathrm{P}=0.32$ for $\mathrm{FFDM}$; and $\mathrm{HR}=2.91,95 \% \mathrm{CI}: 0.41-20.7 ; \mathrm{P}=0.33$ for CSS, data not shown). Unlike RRM1, the ERCC1 status did not predict whether the patients had a survival benefit from platinum- or non-platinum-based regimens.

As the patients with tumors exhibiting high expression of RRM1 also had higher, albeit non-significantly, rates of advanced tumor stage and other known adverse prognostic factors, we evaluated the prognostic value of RRM1 in MVA, which considered tumor stage (II vs. III-IV), hydronephrosis and VI. Although the negative effect of RRM1 was greatest as measured by the HR when including these 4 variables, none of the variables retained statistical significance (Table III).

\section{Discussion}

$\mathrm{BC}$ is a radiosensitive and chemosensitive tumor with $65 \%$ response and $42 \% \mathrm{CR}$ rates following irradiation with 50 Gy (24). Cisplatin-based regimens, which achieve $40-65 \%$ response and $10-20 \%$ CR rates in metastatic disease (8), were also shown to improve survival in patients with localized BC when administered prior to cystectomy or radiation (7). Bladder preservation protocols combining 
Table III. Multivariate analysis.

\begin{tabular}{|c|c|c|c|c|c|c|}
\hline \multirow[b]{2}{*}{ Variables } & \multicolumn{3}{|c|}{ Bladder cancer-specific mortality } & \multicolumn{3}{|c|}{ Distant metastasis } \\
\hline & HR & P-value & $95 \% \mathrm{CI}$ & HR & P-value & $95 \% \mathrm{CI}$ \\
\hline \multicolumn{7}{|c|}{ Tumor stage } \\
\hline II & \multicolumn{3}{|c|}{ Reference } & \multicolumn{3}{|c|}{ Reference } \\
\hline III-IV & 1.5 & 0.63 & $0.31-7.0$ & 1.1 & 0.90 & $0.18-6.9$ \\
\hline \multicolumn{7}{|c|}{ Vascular invasion } \\
\hline No & \multicolumn{3}{|c|}{ Reference } & \multicolumn{3}{|c|}{ Reference } \\
\hline Yes & 1.2 & 0.89 & $0.27-4.8$ & 2.3 & 0.29 & $0.50-10.2$ \\
\hline \multicolumn{7}{|c|}{ Hydronephrosis } \\
\hline No & \multicolumn{3}{|c|}{ Reference } & \multicolumn{3}{|c|}{ Reference } \\
\hline Yes & 1.9 & 0.40 & $0.43-8.4$ & 3.7 & 0.12 & $0.72-18.7$ \\
\hline \multicolumn{7}{|c|}{ RRM1 expression } \\
\hline Low & \multicolumn{3}{|c|}{ Reference } & \multicolumn{3}{|c|}{ Reference } \\
\hline High & 3.0 & 0.15 & $0.69-12.8$ & 1.7 & 0.52 & $0.35-7.8$ \\
\hline
\end{tabular}

HR, hazard ratio; CI, confidence interval; RRM1, ribonucleoside reductase subunit M1.

A

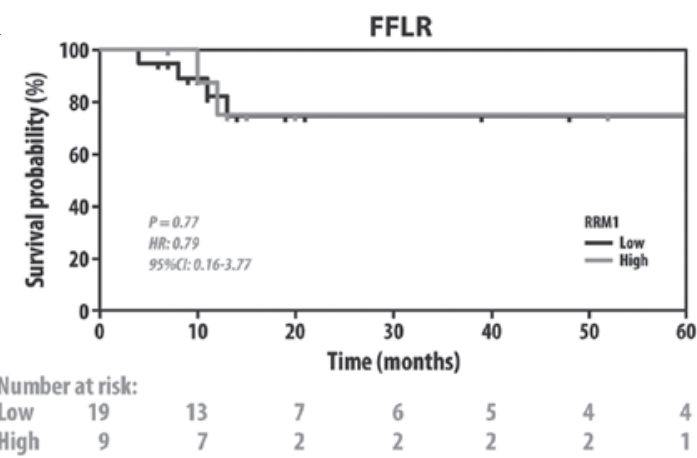

B

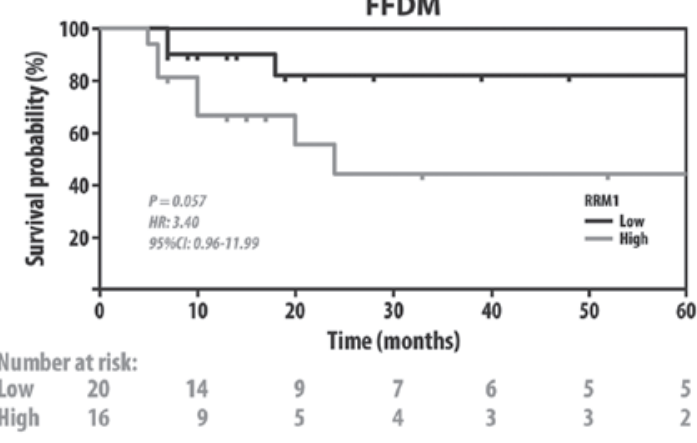

C

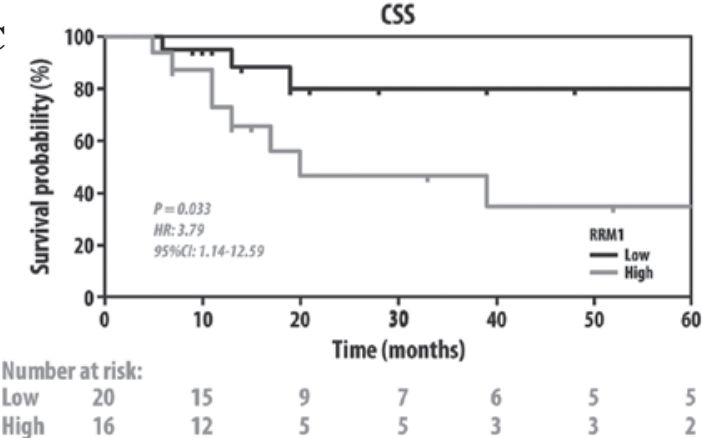

D
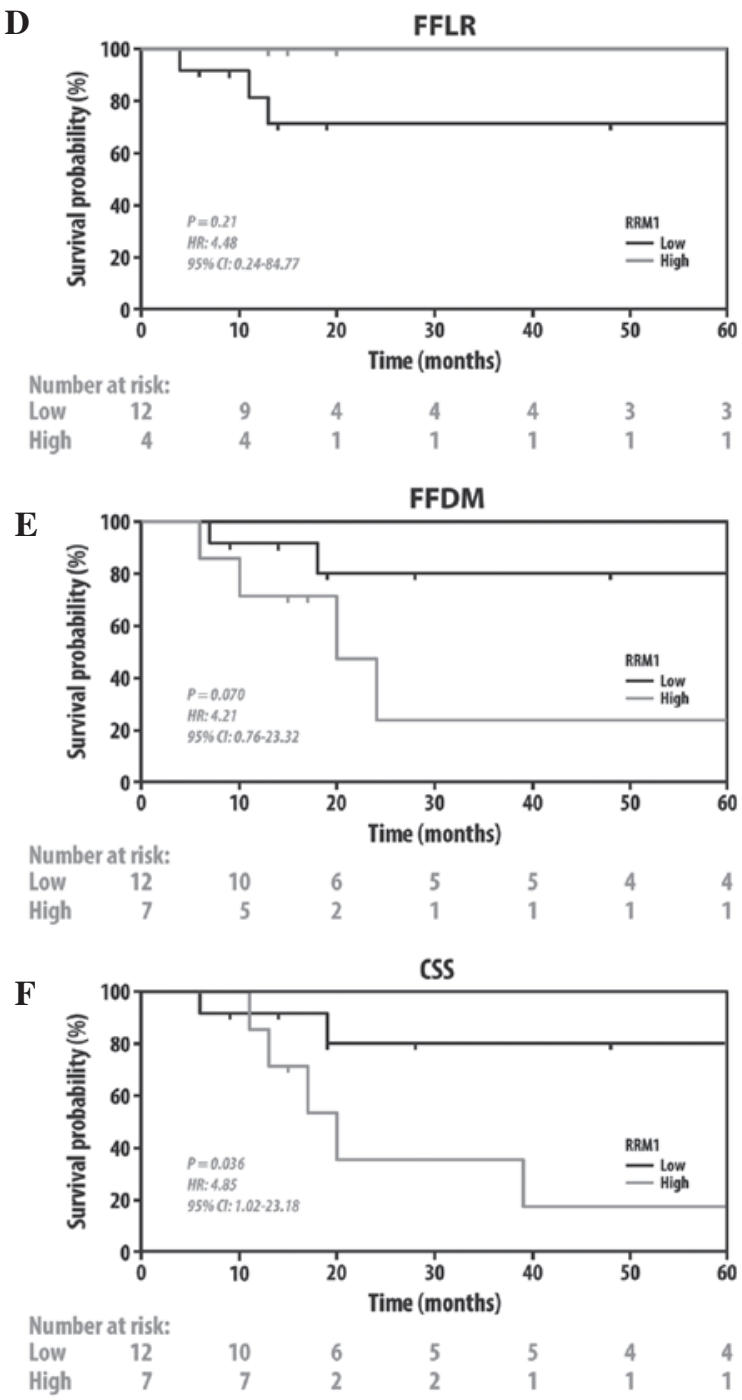

Figure 3. Kaplan-Meier plots of FFLR, FFDM and CSS in (A-C) all the patients; and (D-F) patients treated with gemcitabine-based chemoradiotherapy, grouped by ribonucleoside reductase subunit M1 status. FFLR, freedom from local recurrence; FFDM, freedom from distant metastasis; CSS, cancer-specific survival. 
A

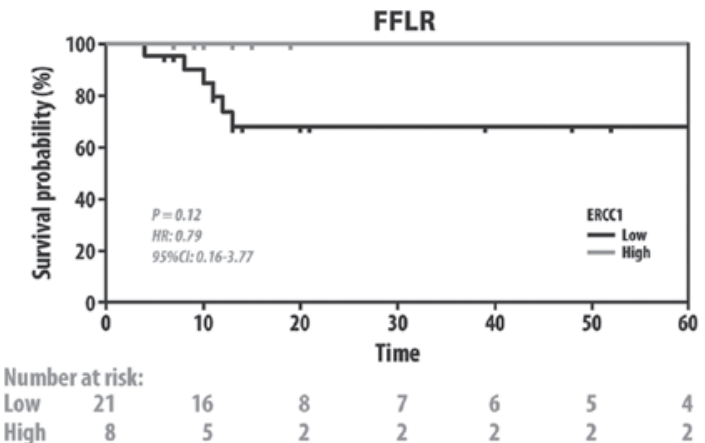

B

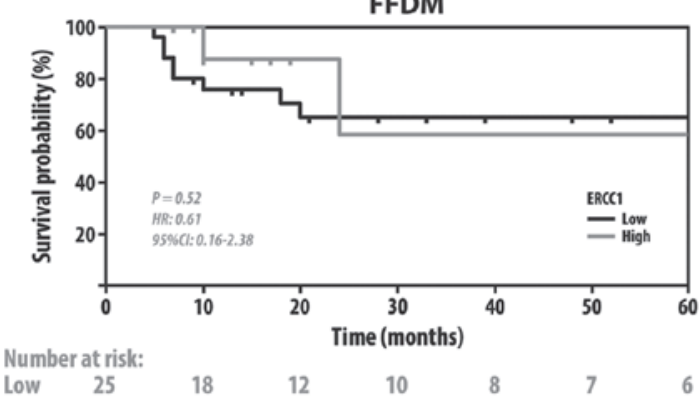

C

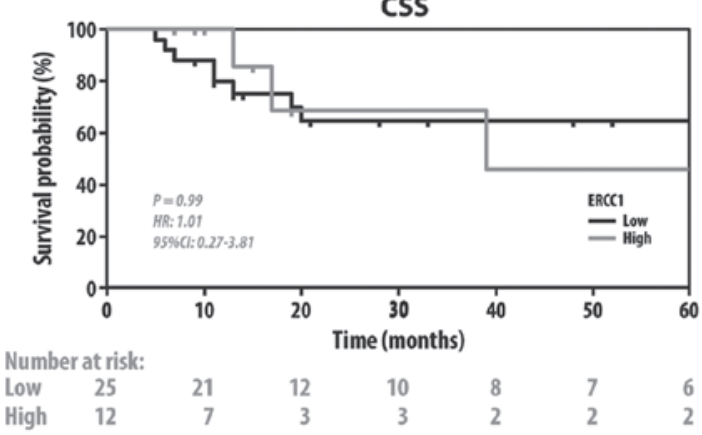

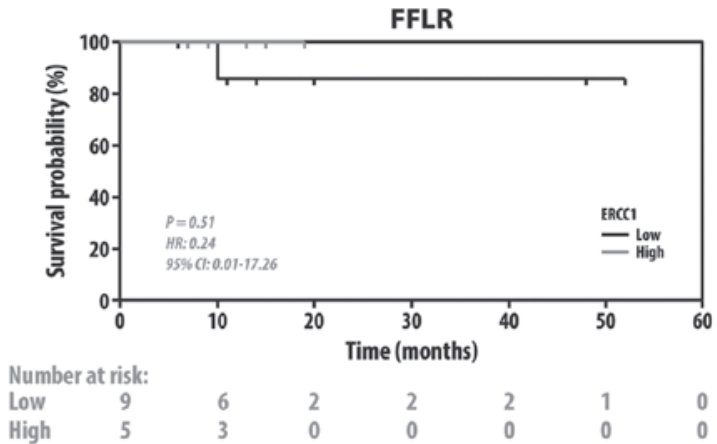

$\mathbf{E}$

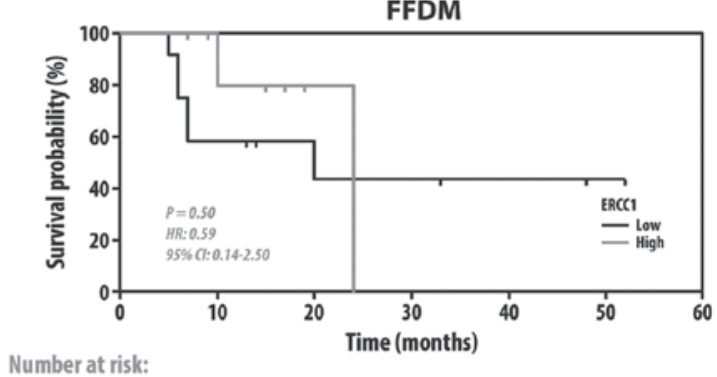

$\mathbf{F}$

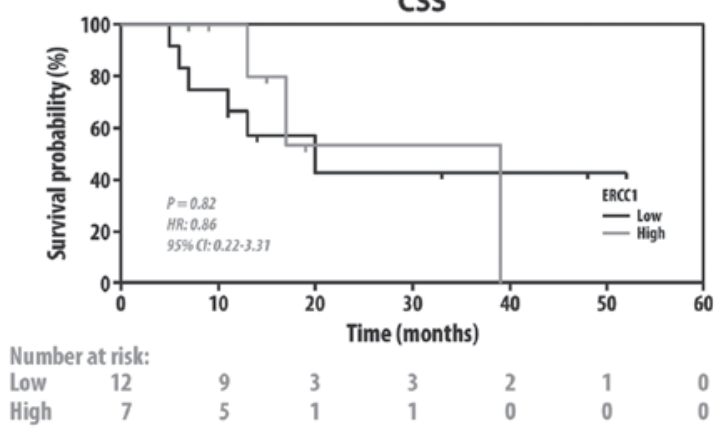

Figure 4. Kaplan-Meier plots of FFLR, FFDM and CSS in (A-C) all the patients and; (D-F) patients treated by platinum-based chemoradiotherapy, grouped by excision repair cross-complementation group 1 status. FFLR, freedom from local recurrence; FFDM, freedom from distant metastasis; CSS, cancer-specific survival.

TURBT, radiotherapy and chemotherapy achieve CR rates of $>70 \%$ and result in survival rates comparable to those of radical surgery $(2,4,23)$. Achieving a $\mathrm{CR}$ is a strong prognostic factor for a favorable clinical outcome and, in the majority of organ-preserving protocols, patients who do not achieve a CR undergo a cystectomy shortly after completing chemoradiotherapy (4). Therefore, a CR following chemoradiotherapy may act as an effective biomarker for the selection of patients for organ preservation treatment, which was previously reported following induction chemotherapy in laryngeal cancer (25), where organ-preserving therapy has become the standard of care. However, patients with BC typically undergo chemoradiotherapy before their pathological response is identified. As a result, a molecular marker, which may help identify patients who are more likely to achieve a CR or guide the selection of chemotherapy regimens, would be of significant value.

In the present study, the predictive and prognostic value of RRM1 and ERCC1 expression was investigated in a group of patients with MIBC treated with platinum- or gemcitabine-based chemoradiotherapy. Our findings demonstrated that low RRM1 expression, which was observed in approximately half of the tumors, was prognostic for CR in patients treated with gemcitabine, but did not affect response in those not receiving gemcitabine. While high RRM1 expression status was also found to be correlated with worse CSS and a trend to worse DM on the univariate analysis, its predictive value was diminished when other adverse clinicopathological characteristics were considered. Nevertheless, the association of CR, DM and CSS with RRM1 expression in patients receiving gemcitabine was consistent with that reported by previous studies investigating other malignancies treated with gemcitabine (9).

RRM1 is the main mechanistic target of gemcitabine and its overexpression is associated with resistance to this drug in vitro and in vivo. In addition to being a key enzyme in the de novo synthesis of nucleotides, RRM1 is also involved in the control of cell proliferation, migration and metastasis (9). The prognostic and predictive properties of RRM1 have been extensively investigated in localized and metastatic NSCLC, where the response rates to gemcitabine-based chemotherapy and survival were found to be inversely correlated with RRM1 expression (26). RRM1 expression was also found to 
be directly correlated with overall survival (OS) in 43 young patients with $\mathrm{BC}$ who underwent cystectomy, in a study that did not record data on LR, DM and the use of chemotherapy and radiotherapy (10). In another study, involving 57 patients with advanced BC treated with gemcitabine-based polychemotherapy, RRM1 expression was not found to be correlated with OS and response $(\mathrm{P}=0.062)$, but was correlated with time-to-disease progression $(\mathrm{P}=0.045)$ (27).

Unlike RRM1, ERCC1 expression was not found to be predictive for the response to platinum-based chemoradiotherapy or prognostic for the benefits from such treatment. There are several available clinical and preclinical studies on the association of ERCC1 expression with resistance to platinum agents $(11,12,17,27-29)$ and radiosensitivity $(15,18,30)$. The ERCC1 gene has 10 exons and generates 4 isoforms by alternative splicing; however, only 1 of these 4 isoforms is actively involved in the repair of platinum-DNA adducts. These isoforms were found to be heterogeneously expressed in NSCLC (31). However, none of the 16 commercially available anti-ERCC 1 antibodies was able to accurately discriminate between the isoforms (31), limiting the usefulness of ERCC1 detection by immunohistochemistry. Moreover, due to the sequence homologies between the isoforms, it is impossible to develop isoform-specific primers for PCR detection of mRNA (31).

Despite the small sample size, which reflects the majority of medically operable patients with MIBC being offered radical surgery $(2,32)$, the present study is one of the very few focused on chemosensitivity markers in BC treated with chemoradiotherapy. Kawashima et al (15) previously evaluated ERCC1 expression in a small and heterogeneous group of 22 patients treated with radiation and platinum agents. To the best of our knowledge, our study is the first to evaluate RRM1 expression in patients undergoing chemoradiotherapy. The limitations of this study lie with its retrospective nature, which did not allow for the control of the clinical characteristics of the patients in the different treatment groups or the heterogeneity of treatment regimens, which reflects the lack of consensus on the most effective treatment for this disease.

In conclusion, our study demonstrated that the expression of RRM1, but not ERCC1, may predict response to gemcitabine-based chemoradiotherapy. As a result, low expression of RRM1 may help identify patients appropriately treated with gemcitabine-based trimodality therapy. Further evaluation of RRM1 as a predictive and prognostic biomarker in larger trials is required, such as the RTOG 0712 trial (33), which randomized patients between gemcitabineand platinum-based regimens concurrent with radiation. The validation of RRM1 may offer a unique molecular means to select treatment regimens for patients with MIBC treated with combined chemotherapy and radiation.

\section{References}

1. Siegel R, Naishadham D and Jemal A: Cancer statistics, 2012. CA Cancer J Clin 62: 10-29, 2012

2. Milosevic M, Gospodarowicz M, Zietman A, et al: Radiotherapy for bladder cancer. Urology 69 (Suppl 1): 80-92, 2007.

3. Efstathiou JA, Bae K, Shipley WU, et al: Late pelvic toxicity after bladder-sparing therapy in patients with invasive bladder cancer: RTOG 89-03, 95-06, 97-06, 99-06. J Clin Oncol 27: 4055-4061, 2009.
4. Efstathiou JA, Spiegel DY, Shipley WU, et al: Long-term outcomes of selective bladder preservation by combined-modality therapy for invasive bladder cancer: the MGH experience. Eur Urol 61: 705-711, 2012.

5. Coppin CM, Gospodarowicz MK, James K, et al: Improved local control of invasive bladder cancer by concurrent cisplatin and preoperative or definitive radiation. The National Cancer Institute of Canada Clinical Trials Group. J Clin Oncol 14: 2901-2907, 1996

6. James ND, Hussain SA, Hall E, et al: Radiotherapy with or without chemotherapy in muscle-invasive bladder cancer. N Engl J Med 366: 1477-1488, 2012.

7. International Collaboration of Trialists, Medical Research Council Advanced Bladder Cancer Working Party, European Organisation for Research and Treatment of Cancer Genito-Urinary Tract Cancer Group, et al: International phase III trial assessing neoadjuvant cisplatin, methotrexate, and vinblastine chemotherapy for muscle-invasive bladder cancer: long-term results of the BA06 30894 trial. J Clin Oncol 29: 2171-2177, 2011.

8. Sternberg CN, Donat SM, Bellmunt J, et al: Chemotherapy for bladder cancer: treatment guidelines for neoadjuvant chemotherapy, bladder preservation, adjuvant chemotherapy, and metastatic cancer. Urology 69 (Suppl 1): 62-79, 2007.

9. Jordheim LP, Seve P, Tredan O and Dumontet C: The ribonucleotide reductase large subunit (RRM1) as a predictive factor in patients with cancer. Lancet Oncol 12: 693-702, 2011.

10. Harshman LC, Bepler G, Zheng Z, Higgins JP, Allen GI and Srinivas S: Ribonucleotide reductase subunit M1 expression in resectable, muscle-invasive urothelial cancer correlates with survival in younger patients. BJU Int 106: 1805-1811, 2010.

11. Zheng Z, Chen T, Li X, Haura E, Sharma A and Bepler G: DNA synthesis and repair genes RRM1 and ERCC1 in lung cancer. N Engl J Med 356: 800-808, 2007.

12. Gossage L and Madhusudan S: Current status of excision repair cross complementing-group 1 (ERCC1) in cancer. Cancer Treat Rev 33: 565-577, 2007.

13. Kim KH, Do IG, Kim HS, et al: Excision repair cross-complementation group 1 (ERCC1) expression in advanced urothelial carcinoma patients receiving cisplatin-based chemotherapy. APMIS 118: 941-948, 2010.

14. Sun JM, Sung JY, Park SH, et al: ERCC1 as a biomarker for bladder cancer patients likely to benefit from adjuvant chemotherapy. BMC Cancer 12: 187, 2012.

15. Kawashima A, Nakayama M, Kakuta Y, et al: Excision repair cross-complementing group 1 may predict the efficacy of chemoradiation therapy for muscle-invasive bladder cancer. Clin Cancer Res 17: 2561-2569, 2011.

16. Vilmar A, Garcia-Foncillas J, Huarriz M, Santoni-Rugiu E and Sorensen JB: RT-PCR versus immunohistochemistry for correlation and quantification of ERCC1, BRCA1, TUBB3 and RRM1 in NSCLC. Lung Cancer 75: 306-312, 2012.

17. Ozcan MF, Dizdar O, Dincer N, et al: Low ERCC1 expression is associated with prolonged survival in patients with bladder cancer receiving platinum-based neoadjuvant chemotherapy. Urol Oncol 31: 1709-1715, 2012.

18. Ahmad A, Robinson AR, Duensing A, et al: ERCC1-XPF endonuclease facilitates DNA double-strand break repair. Mol Cell Biol 28: 5082-5092, 2008

19. Nocito A, Kononen J, Kallioniemi OP and Sauter G: Tissue microarrays (TMAs) for high-throughput molecular pathology research. Int J Cancer 94: 1-5, 2001.

20. Smith DC, Mackler NJ, Dunn RL, et al: Phase II trial of paclitaxel, carboplatin and gemcitabine in patients with locally advanced carcinoma of the bladder. J Urol 180: 2384-2388, 2008.

21. Higano CS, Tangen CM, Sakr WA, et al; Southwest Oncology Group Trial 8733: Treatment options for muscle-invasive urothelial cancer for patients who were not eligible for cystectomy or neoadjuvant chemotherapy with methotrexate, vinblastine, doxorubicin, and cisplatin: report of Southwest Oncology Group Trial 8733. Cancer 112: 2181-2187, 2008.

22. Kent E, Sandler H, Montie J, et al: Combined-modality therapy with gemcitabine and radiotherapy as a bladder preservation strategy: results of a phase I trial. J Clin Oncol 22: 2540-2545, 2004.

23. Shipley WU,Zietman AL, Kaufman DS, Coen JJ and Sandler HM: Selective bladder preservation by trimodality therapy for patients with muscularis propria-invasive bladder cancer and who are cystectomy candidates - the Massachusetts General Hospital and Radiation Therapy Oncology Group experiences. Semin Radiat Oncol 15: 36-41, 2005. 
24. Pollack A, Zagars GK, Dinney CP, et al: Preoperative radiotherapy for muscle-invasive bladder carcinoma. Long term follow-up and prognostic factors for 338 patients. Cancer 74 : 2819-2827, 1994

25. No authors listed: Induction chemotherapy plus radiation compared with surgery plus radiation in patients with advanced laryngeal cancer. The Department of Veterans Affairs Laryngeal Cancer Study Group. N Engl J Med 324: 1685-1690, 1991.

26. Ceppi P, Volante M, Novello S, et al: ERCC1 and RRM1 gene expressions but not EGFR are predictive of shorter survival in advanced non-small-cell lung cancer treated with cisplatin and gemcitabine. Ann Oncol 17: 1818-1825, 2006.

27. Bellmunt J, Paz-Ares L, Cuello M, et al: Gene expression of ERCC1 as a novel prognostic marker in advanced bladder cancer patients receiving cisplatin-based chemotherapy. Ann Oncol 18: 522-528, 2007.

28. Kawashima A, Takayama $\mathrm{H}$ and Tsujimura A: A review of ERCC1 gene in bladder cancer: implications for carcinogenesis and resistance to chemoradiotherapy. Adv Urol 2012: 812398, 2012.
29. Simon GR, Schell MJ, Begum M, et al: Preliminary indication of survival benefit from ERCC1 and RRM1-tailored chemotherapy in patients with advanced nonsmall cell lung cancer: evidence from an individual patient analysis. Cancer 118: 2525-2531, 2012.

30. Liu ZG, Chen HY, Cheng JJ, Chen ZP, Li XN and Xia YF: Relationship between methylation status of ERCC1 promoter and radiosensitivity in glioma cell lines. Cell Biol Int 33: 1111-1117, 2009.

31. Friboulet L, Olaussen KA, Pignon JP, et al: ERCC1 isoform expression and DNA repair in non-small-cell lung cancer. N Engl J Med 368: 1101-1110, 2013.

32. Fedeli U, Fedewa SA and Ward EM: Treatment of muscle invasive bladder cancer: evidence from the National Cancer Database, 2003 to 2007. J Urol 185: 72-78, 2011

33. RTOG 0712 Protocol Information. February 28, 2012: http:// www.rtog.org/ClinicalTrials/ProtocolTable/StudyDetails aspx?study=0712. Accessed December 17, 2013. 